\title{
ARTICLE OPEN Realizing universal quantum gates with topological bases in quantum-simulated superconducting chains
}

\author{
Yong $\mathrm{Hu}^{1,2}$, Y. X. Zhao ${ }^{2}$, Zheng-Yuan Xue ${ }^{3,2}$ and Z. D. Wang ${ }^{2}$
}

One-dimensional time-reversal invariant topological superconducting wires of the symmetry class DIII exhibit exotic physics which can be exploited to realize the set of universal operations in topological quantum computing. However, the verification of DIII-class physics in conventional condensed matter materials is highly nontrivial due to realistic constraints. Here we propose a symmetryprotected hard-core boson simulator of the one-dimensional DIII topological superconductor. By using the developed dispersive dynamic modulation approach, not only the faithful simulation of this new type of spinful superconducting chains is achieved, but also a set of universal quantum gates can be realized with the computational basis formed by the degenerate ground states that are topologically protected against random local perturbations. Physical implementation of our scheme based on a Josephson quantum circuit is presented, where our detailed analysis pinpoints that this scheme is experimentally feasible with the state-ofthe-art technology.

npj Quantum Information (2017)3:8; doi:10.1038/s41534-017-0009-3

\section{INTRODUCTION}

Topological band theory has become the focus of recent research due to its significant importance in fundamental physics and potential applications in novel devices. ${ }^{1-3}$ In each spatial dimension, topological insulators and topological superconductors can well be classified in terms of the presence or absence of time-reversal symmetry (TRS) and particle-hole symmetry. ${ }^{4}, 5$ Typical examples include the integer quantum Hall effect ${ }^{6}$ belonging to the $A$ class of $2 \mathrm{D}$ and the Kitaev chain belonging to the $\mathrm{D}$ class of $1 \mathrm{D} .^{7}$ Among the various symmetry-protected topological phases, increasing research interests have been drawn to the theoretical and experimental studies of 1D topological superconducting wires in the symmetry class DIII. ${ }^{8-14}$ In contrast to the Kitaev chain in the class D with one Majorana zero mode (MZM) at each end and two-fold degenerate ground states in its $\mathbb{Z}_{2}$ topological phase, ${ }^{7}$ the DIII superconductor wire in the $\mathbb{Z}_{2}^{(2)}$ topological phase possesses the TRS, and has a Kramers doublet of MZMs at each end and four-fold degenerate ground states. ${ }^{15-17}$ Moreover, the DIII superconductor wire exhibits an impactful response to an effective magnetic field and long-range spincorrelation at the two ends with the fixed fermionic parity, which can be utilized to realize universal quantum operations of qubits that are topologically protected against random local perturbations. ${ }^{9}, 12,13$ However, despite the extensive theoretical efforts, still the 1D TRS-associated $\mathbb{Z}_{2}^{(2)}$ topological phase has not been tested experimentally in electronic systems as it is limited by realistic reasons, e.g. the constraints of materials, the lack of controllability, and the co-existing complicated mechanisms.

On the other hand, it has been indicated that a kind of onedimensional (1D) hard-core boson (HCB) chain may be able to simulate fermionic physics, ${ }^{18}$ with interesting phenomena being investigated including the pursuit of Majorana fermions in the
Kitaev model, ${ }^{19-21}$ the string breaking dynamics of quark pairs, ${ }^{22}$ the electron-electron scattering, ${ }^{23}$ and the delocalization of Dirac fermions in disordered 1D wires. ${ }^{24}$ However, methods in the existing studies are restricted to spinless fermions, where the forms of the concerned Hamiltonians remain unchanged during the bosonization. When bosonizing a spinful 1D fermionic system, an exotic particle-density-dependent $U(1)$ gage phase factor emerges from fermionic statistics among fermions with opposite spin orientations, leading to one of main challenges in the simulation of the spinful DIII models.

In this paper, we reveal unambiguously that a 1D HCB lattice can be exploited to faithfully simulate the proposed DIII model which realizes the nontrivial $\mathbb{Z}_{2}$ topological phase. ${ }^{12}$ Most remarkably, with the odd-parity Kramers doublet ground states being used as the basis of topological qubits, we demonstrate for the first time that a set of universal quantum gates can in principle be achieved using these topological bases with the help of this HCB architecture, where we develop a dispersive dynamic modulation (DDM) approach to mediate the exotic $U(1)$ gage field configuration in the bosonized DIII model. Moreover, we propose a physical implementation scheme based on superconducting quantum circuit (SQC). ${ }^{25}, 26$ Taking the advantages advantage of flexibility and scalability of $\mathrm{SQC}_{1}{ }^{27,}{ }^{28}$ we encode $\mathrm{HCBs}$ by superconducting transmon qubits and design the nontrivial coupling through the inductive connection between transmon qubits. ${ }^{29-32}$ Our estimation also implies that the effective coupling strengths can be several orders larger than that corresponding to the coherence time the decoherence rates of the transmon qubits, ${ }^{33}, 34$ and therefore the proposed topological operations may be tested with the current level of technology.

\footnotetext{
${ }^{1}$ School of Physics, Huazhong University of Science and Technology, Wuhan 430074, China; ${ }^{2}$ Department of Physics and Center of Theoretical and Computational Physics, The

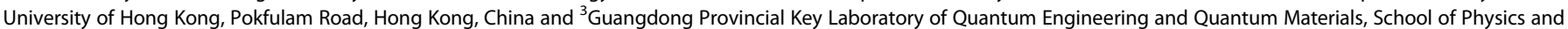
Telecommunication Engineering, South China Normal University, Guangzhou 510006, China Correspondence: Yong Hu (huyong@mail.hust.edu.cn)
}

Received: 25 February 2016 Revised: 18 January 2017 Accepted: 26 January 2017 Published online: 06 March 2017 


\section{RESULTS}

The HCB realization of DIII model

An intriguing model Hamiltonian of fermionic 1D topological superconductor in the class DIII reads, ${ }^{12}$

$H_{\text {DIII }}=\sum_{j a}\left(-w c_{j, a}^{\dagger} c_{j+1, a}-i \Delta c_{j+1, a} c_{j, \bar{\alpha}}+\right.$ h.c. $)-\mu\left(c_{j, a}^{\dagger} c_{j, a}-1\right)$

where $c_{j, a}^{\dagger}$ is the creation operator of the spin- $a$ fermion on the $j$ th site with $a=\uparrow, \downarrow, w$ is the real-value tight-binding hopping strength, $\mu$ is the chemical potential, $\Delta$ is the real-value real amplitude of $\mathrm{p}$-wave pairing parameter, and $\bar{a}$ denotes the spin component opposite to $a$. Under the basis transformation $a_{j}=$ $e^{-i \pi / 4}\left(c_{j, \uparrow}+c_{j, \downarrow}\right) / \sqrt{2}$ and $\bar{a}_{j}=e^{-i \pi / 4}\left(c_{j, \uparrow}-c_{j, \downarrow}\right) / \sqrt{2}, H_{\text {DIII }}$ can be rewritten as

$H_{\text {DIII }}=H+\bar{H}$

with

$H=\sum_{j}\left(-w a_{j}^{\dagger} a_{j+1}+\Delta a_{j+1} a_{j}+\right.$ h.c. $)-\mu\left(a_{j}^{\dagger} a_{j}-1 / 2\right)$
$\bar{H}=\sum_{j}\left(-w \bar{a}_{j}^{\dagger} \bar{a}_{j+1}-\Delta \bar{a}_{j+1} \bar{a}_{j}+\right.$ h.c. $)-\mu\left(\bar{a}_{j}^{\dagger} \bar{a}_{j}-1 / 2\right)$

i.e., $H_{\text {DIII }}$ is decoupled into two Kitaev chains differed by the signs of their superconducting order parameters (hereafter we denote the quantities related with the (+)-chain as $A$ and their counterparts for the (-)-chain as $\bar{A})$. Although the topology-preserved simulation of a standard Kitaev model with $\mathrm{HCBs}$ has been proposed, $^{19-21}$ to simulate $H_{\text {DIII }}$ with HCBs is much more challenging than the separate simulations of $\mathrm{H}^{+}$and $\mathrm{H}^{-}$by two independent $\mathrm{HCB}$ chains, because such straightforward scenario cannot result in the fermionic anti-commutation relation of interspecies. For this, here we exploit a quasi-1D HCB chain shown in Fig. 1(a), where the upper/lower array plays the role of the +/chain, respectively. The DIII model (2) can be bosonized as

$$
\begin{aligned}
& H=\sum_{j}\left(-w \bar{P}_{j} b_{j}^{\dagger} b_{j+1}+\Delta \bar{P}_{j} b_{j+1} b_{j}+\text { h.c. }\right)-\mu\left(b_{j}^{\dagger} b_{j}-\frac{1}{2}\right) \\
& \bar{H}=\sum_{j}\left(-w P_{j+1} \bar{b}_{j}^{\dagger} \bar{b}_{j+1}-\Delta P_{j+1} \bar{b}_{j+1} \bar{b}_{j}+\text { h.c. }\right)-\mu\left(\bar{b}_{j}^{\dagger} \bar{b}_{j}-\frac{1}{2}\right)
\end{aligned}
$$

with the bosonization along the zig-zag path in Fig. 1(a) being given by $b_{j}=a_{j} \prod_{s<j} P_{s} \prod_{s<j} \bar{P}_{s}$ and $\bar{b}_{j}=\bar{a}_{j} \prod_{s<j+1} P_{s} \prod_{s<j} \bar{P}_{s}$. Here $b_{j}\left(\bar{b}_{j}\right)$ is the annihilation operators of the $j$ th $\mathrm{HCB}$ on the $+(-)$ chain, $\quad P_{s}=\exp \left(-i \pi a_{s}^{\dagger} a_{s}\right)\left[=\exp \left(-i \pi b_{s}^{\dagger} b_{s}\right)\right], \quad$ and $\bar{P}_{s}=\exp \left(-i \pi \bar{a}_{s}^{\dagger} \bar{a}_{s}\right)$. Although this is a nonlocal transformation, the locality of operators of physical noises (or random perturbations) is preserved, and so does the topological stability (This bosonization inherits topological protection against local fluctuations seen from the original fermionic system of Eq. (2), because the locality of the three forms of physical perturbations $\left(b_{j}^{\dagger} b_{j+1}, b_{j+1} b_{j}, b_{j}^{\dagger} b_{j}\right)$ in the simulated bosonic system remains unchanged after fermionization. To be concrete in other words, if the transformed terms, which correspond to possible random local (perturbation) terms in the simulated system, are still local ones in the original fermonic picture of topological model after application of the non-local transformation, one can deduce that the simulated system is also topologically protected.). It is noticed that the fermionic anti-commutation relation has been converted to the gage field factors after bosonization, which in one chain is given by the particle density of the other chain in a site-wise sense.

To implement the bosonization version of the DIII model (3), we set that the HCBs on the upper/lower array of Fig. 1(a) have energy splits $\Omega / \bar{\Omega}$ with $\delta=\Omega-\bar{\Omega} \sim 0.3 \Omega$, and the inter-HCB coupling takes the nearest-neighbor form $H^{L}=\sum_{j} \mathcal{H}_{j}^{L}+\overline{\mathcal{H}}_{j}^{L}$ with $\mathcal{H}_{j}^{L}=$ $V_{j}(t)\left(b_{j}^{\dagger}+b_{j}\right)\left(\bar{b}_{j}^{\dagger}+\bar{b}_{j}\right)$ and $\overline{\mathcal{H}}_{j}^{L}=\bar{V}_{j}(t)\left(\bar{b}_{j}^{\dagger}+\bar{b}_{j}\right)\left(b_{j+1}^{\dagger}+b_{j+1}\right)$ where $\left|V_{j}(t)\right|,\left|\bar{V}_{j}(t)\right| \simeq 10^{-2} \delta$ are the coupling strengths which can be modulated harmonically and in situ. We further introduce a dispersive energy threshold $\eta \simeq 10^{-1} \delta$ such that $\left|V_{j}(t)\right|,\left|\bar{V}_{j}(t)\right| \ll$ $\eta \ll \delta$ and modulate $V_{j}(t)$ and $\bar{V}_{j}(t)$ as

$$
\begin{aligned}
& V_{j}(t)=2 s_{j} \cos (\eta-\delta) t+2 q_{j} \cos (\eta-\varepsilon) t \\
& \bar{V}_{j}(t)=2 \bar{s}_{j} \cos (\eta-\delta) t+2 \bar{q}_{j} \cos (\eta+\varepsilon) t
\end{aligned}
$$

with $\varepsilon=\Omega+\bar{\Omega}$. Based on the parameter choice

$$
\begin{aligned}
& s_{j}=(-1)^{j-1} s, q_{j}=(-1)^{j-1} q \\
& \bar{s}_{j}=(-1)^{j-1} \bar{s}, \bar{q}_{j}=(-1)^{j-1} \bar{q}, \bar{q} / \bar{s}=-q / s
\end{aligned}
$$

we can directly reproduce the hopping and pairing terms in Eq. (3) with $w=-s \bar{s} / \eta$ and $\Delta=s \bar{q} / \eta$ from the dispersive coupling between $\sum_{j} \mathcal{H}_{j}^{L}$ and $\sum_{j} \overline{\mathcal{H}}_{j}^{L}$ (For detailed derivation we refer to Methods). The obtained amplitudes of $w$ and $\Delta$ can be independently tuned in the range $\left[10^{-3}, 10^{-2}\right] \delta$ by the parameters $(s, q, \bar{s}, \bar{q})$. In addition, the chemical potential term in Eq. (3) can be produced by adding an on-site dispersive radiation $H^{D}=\sum_{j} D_{j}(t)\left(b_{j}^{\dagger}+b_{j}\right)+\bar{D}_{j}(t)\left(\bar{b}_{j}^{\dagger}+\bar{b}_{j}\right)$. A convenient choice of $D_{j}(t)$ (and similarly $\left.\bar{D}_{j}(t)\right)$ is $D_{j}(t)=d_{j}\left[e^{j(\Omega \pm 3 n)}+e^{-i(\Omega \pm 3 \eta)}\right]$ which results in the a.c. Stark shift $\pm d_{j}^{2} b_{j}^{\dagger} b_{j} / 3 \eta$. The $3 \eta$ setting of the radiation frequency is to avoid the crosstalk between $H^{D}$ and $H^{L}$, and the choice of the $3 \eta$ sign depends on whether positive or negative correction of the chemical potential $\mu$ is needed.

\section{MZMs and the universal quantum gates}

The region $|w|>|\mu / 2|$ can be identified as the topological phase where the nontrivial MZMs emerge. ${ }^{7}$ Especially, we focus on the ideal case $w=\Delta$ and $\mu=0$ where the DIII model (2) can simply be reduced to

$H_{\text {DIII }}=\sum_{j} i w\left(\gamma_{j, B} \gamma_{j+1, A}+\bar{\gamma}_{j, B} \bar{\gamma}_{j+1, A}\right)$,

with Majorana operators $\quad \gamma_{j, A}=i\left(a_{j}-a_{j}^{\dagger}\right), \quad \gamma_{j, B}=a_{j}+a_{j}^{\dagger}$, $\bar{\gamma}_{j, A}=\bar{a}_{j}+\bar{a}_{j}^{\dagger}$, and $\bar{\gamma}_{j, B}=-i\left(\bar{a}_{j}-\bar{a}_{j}^{\dagger}\right)$. The Hamiltonian is fully diagonalized through the pairing of $\gamma_{j, B}$ and $\gamma_{j+1, A}$ (and simultaneously $\bar{\gamma}_{j, B}$ and $\left.\bar{\gamma}_{j+1, A}\right)$ in the bulk, leaving four MZMs $\gamma_{A}=\gamma_{1, A}$, $\bar{\gamma}_{A}=\bar{\gamma}_{1, A}, \gamma_{B}=\gamma_{N, B}$ and $\bar{\gamma}_{B}=\bar{\gamma}_{N, B}$ unpaired at the two ends ( $N$ being the length of the DIII chain). The ground states are thus four-fold degenerate with a basis $\{|1\rangle|\overline{1}\rangle,|0\rangle|\overline{1}\rangle,|1\rangle|\overline{0}\rangle,|0\rangle|\overline{0}\rangle\}$ satisfying $\left.-i \gamma_{A}\right\rangle_{B}|0,1\rangle= \pm|0,1\rangle$ and $-i \bar{\gamma}_{A} \bar{\gamma}_{B}|\overline{0}, \overline{1}\rangle= \pm|\overline{0}, \overline{1}\rangle$. Here we choose the ground subspace spanned by $|\bullet\rangle=|0\rangle|\overline{1}\rangle$ and $|\times\rangle=$ $|1\rangle|\overline{0}\rangle$ as a topological qubit. Such subspace has the odd parity distinguishing it from the other ground states. For comparison, we recall that the ground state of the Kitaev model with only one MZM at each end have the opposite (even) fermionic parity.

It is shown that a single Kitaev chain can be minimally simulated by a 3-HCB array. ${ }^{20}$ Therefore, for an easy experimental setup but without loss of generality, we consider the 12-HCB lattice sketched in Fig. 1(b) to demonstrate the universal quantum operations in the topological bases. We notice that two DIII chains can be constructed by "cutting" the proposed HCB array into two pieces (i.e. by tuning $\bar{s}_{3}=\bar{q}_{3}=0$ and $q_{4}=0$. Note that turning on or off these coupling parameters does not have any impact on the total ground state energy as MZMs have no contributions to the energy, namely, the total energy is conserved under these operations in the ground state, with the TRS being also preserved.) These two subchains, labeled by $L$ and $R$, have consequently 8 emerged MZMs at the ends (4 for each subchain, see Fig. 1(b)). For each subchain, the previously established formalism of defining topological qubits can still be exploited. For universal single qubit operation, we take the left subchain as an example by considering $H_{\mathrm{S}}=\mathbf{B} \cdot \hat{\mathbf{S}}^{L}$ with $\mathbf{B}$ as an effective magnetic field, which is very weak compared with the bulk gap, and $\hat{\mathbf{s}}^{L}=\sum_{j=1}^{3}\left(a_{j}^{\dagger}, \bar{a}_{j}^{\dagger}\right) \hat{\sigma}\left(a_{j}, \bar{a}_{j}\right)^{T}$. It has been shown that $\hat{\mathbf{S}}^{L}$ can be regarded as an effective single spin operator acting on the left 
a)

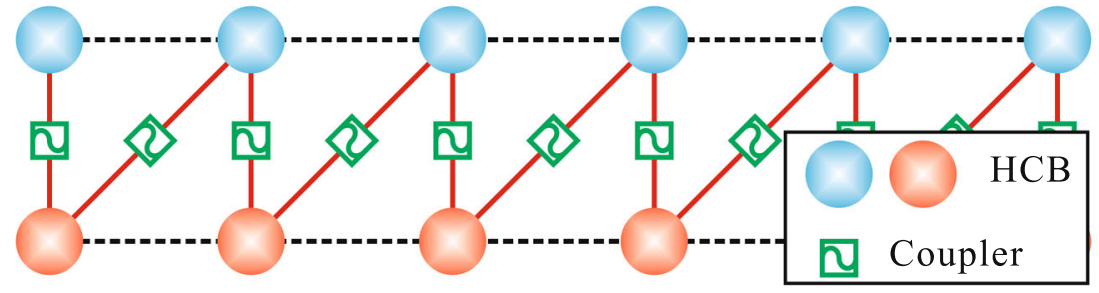

b)

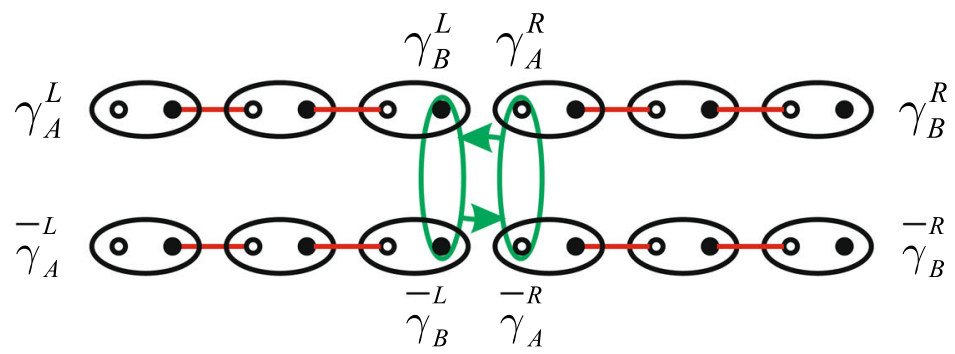

c)

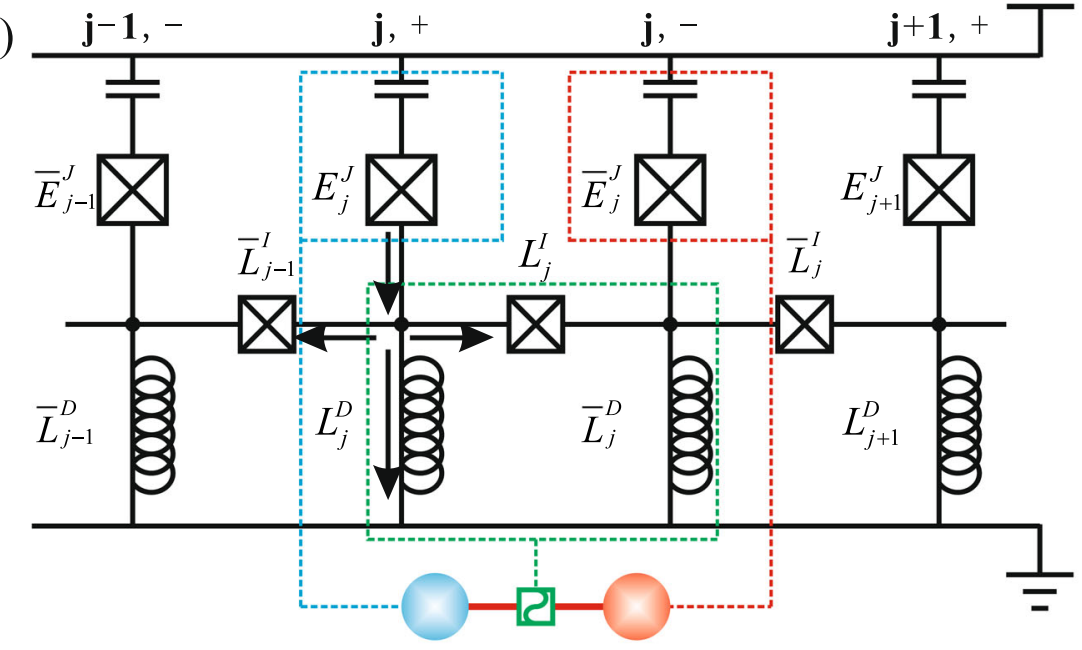

Fig. 1 a Schematic view of the 1-D HCB simulator of $H_{\text {DIII. }}$ The solid red line describes the path along which the HCB sites are physically coupled and the bosonization is performed. Through the DDM of the nearest neighbor coupling (the green wavelet boxes) the effective intraspecies hopping and pairing (the black dashed lines) can be induced. The 12-HCB lattice shown is minimal for the demonstration of universal quantum operations. b Realization of nontrivial two-qubit gate in the 12-HCB lattice. In the situation $w=\Delta$ and $\mu=0$, each HCB site (the ellipse) can be decomposed into two Majorana modes (the filled and the hollow dots) pairing in the bulk. Two topological qubits can be prepared by " cutting" the whole DIII chain into two pieces. Through the modulation of $(3,-) \leftrightarrow(4,+)$ coupling, the effective $\gamma_{B}^{L} \gamma_{A}^{R}+\bar{\gamma}_{B}^{L} \bar{\gamma}_{A}^{R}$ coupling can be induced. c Schematic plot of the array of coupled transmon qubits

topological qubit, with $|\cdot\rangle^{L}$ and $|x\rangle^{L}$ being the two eigenstates of $\mathbf{S}_{z}^{L}{ }^{12}$ Thus through the control of $\mathbf{B}$ the universal single-qubit operation can be achieved. Notably, since the qubits are constructed by the ground states of the whole system, only the global change of the direction of B may manipulate them, while random local variations are actually irrelevant (Note that the TRS is weakly broken during a generic adiabatic operation of the single qubit gate, but the operation, similar to the case of the Kitaev chain, is still topologically protected by the particle-hole symmetry of the superconductor, such that this type of gate is no doubt a symmetry-protected topological one unless the superconducting gap vanishes). The $\mathbf{S}_{x}^{L}$ rotation can be implemented by the resonant dynamic modulation approach: for each $(j,+) \leftrightarrow(j,-)$ link, we add a resonant tone $V_{j}^{R X}(t)=2 B_{x}(t) \cos \delta t$ to $V_{j}(t)$ with $B_{x}(t)$ the slow-varying (adiabatic) pulse amplitude satisfying $B_{x}\left(t_{i}\right)=$ $B_{x}\left(t_{f}\right)=0$ to induce the resonant inter-species hopping $\sum_{j=1}^{3} B_{x}(t)\left(b_{j}^{\dagger} \bar{b}_{j}+b_{j} \bar{b}_{j}^{\dagger}\right)$ which is equivalent to $H_{S x}=B_{x}(t) \mathbf{S}_{x}^{L}$ in the fermionic picture. The rotation $\exp \left(-i \theta \mathbf{S}_{x}^{L}\right)$ with $\theta=\int_{t_{i}}^{t_{f}} B_{x} d t$ can be realized after $t_{f}$. The $\mathbf{S}_{y}^{L}$ rotation can be similarly realized by changing the initial phase of the resonant dynamic modulation as $V_{j}^{R Y}(t)=2 B_{y}(t) \cos (\delta t-\pi / 2)$.
To implement the nontrivial inter-qubit quantum gate, we modulate $\bar{V}_{3}(t)$ as $\bar{V}_{3}(t)=2 \bar{h}(t) \cos (\eta-\delta) t$ to induce the effective $(3,+) \leftrightarrow(4,+)$ and $(3,-) \leftrightarrow(4,-)$ hopping which can be written as

$H_{M}^{T}=-i \frac{S_{M}}{2}\left(\gamma_{B}^{L} \gamma_{A}^{R}+\bar{\gamma}_{B}^{L} \bar{\gamma}_{A}^{R}\right)$

with $s_{M}=s \bar{h}(t) / \eta$. The coupling strength $s_{M}$ can be controlled by the slow-varying envelope $\bar{h}(t)$ such that $\left|s_{M}\right| \ll \Delta, s_{M}(0)=s_{M}\left(t_{f}\right)=$ 0 , and $\int_{t_{i}}^{t_{f}} s_{M} d t=\pi$. Consequently, we get an unitary transformation $U=-\gamma_{B}^{L} Y_{A}^{R} \bar{Y}_{B}^{L} \bar{Y}_{A}^{R}$ transforming the four basis states as

$$
\begin{aligned}
& |\bullet\rangle^{L}|\bullet\rangle^{R} \rightarrow|x\rangle^{L}|x\rangle^{R}, \quad|x\rangle^{L}|\bullet\rangle^{R} \rightarrow-|\bullet\rangle^{L}|x\rangle^{R}, \\
& |\bullet\rangle^{L}|x\rangle^{R} \rightarrow-|x\rangle^{L}|\bullet\rangle^{R}, \quad|x\rangle^{L}|x\rangle^{R} \rightarrow|\bullet\rangle^{L}|\bullet\rangle^{R},
\end{aligned}
$$

i.e., $U$ acts as a nontrivial two-qubit gate $-\mathbf{S}_{y}^{L} \otimes \mathbf{S}_{y}^{R}$ which can be exploited to achieve a set of universal quantum gates in combination with the single-qubit gates mentioned previously. 
Physical implementation with superconducting circuits

We now elaborate in detail how to implement the present theoretical scheme by SQCs. For the site $(j,+)$ we consider a superconducting transmon qubit consisting of a superconducting quantum interference device (SQUID) with effective Josephson energy $E_{j}^{j}$ shunted by a large capacitance $C_{j r}{ }^{29,} 30$ as shown in Fig. 1(c). The two characteristic energy scales of the transmon qubit are its anharmonicity $E_{j}^{C}=e^{2} / 2 C_{j}$ and its lowest level splitting $\Omega_{j} \simeq \sqrt{8 E_{j}^{C} E_{j}^{\jmath}}$. Here we choose $j$-independent $\Omega_{j} / 2 \pi=\Omega /$ $2 \pi=10 \mathrm{GHz}$ and $E_{j}^{C} / 2 \pi=E^{C} / 2 \pi \simeq 0.75 \mathrm{GHz}$. The HCB sites on the ' - ' chain can be similarly constructed with $\bar{\Omega}_{j} / 2 \pi=\bar{\Omega} / 2 \pi=$ $7.5 \mathrm{GHz}$ and $\bar{E}_{j}^{C} \simeq E_{j}^{C}$. Due to the large anharmonicity, the presence of the higher levels of the transmons can only slightly modify the effective parameters derived below and the transmons can be consequently modeled by the two-level HCB Hamiltonian $H^{\mathrm{HCB}}=\sum_{j} \Omega b_{j}^{\dagger} b_{j}+\bar{\Omega}_{b}^{\dagger} \bar{b}_{j}$. For the links between neighboring qubits, we exploit the current dividing mechanism recently studied in experiments. ${ }^{31,32}$ The transmon qubit design is slightly modified by introducing a small grounding inductance $L_{j}^{D} \simeq L_{j}^{J} / 2$ with $L_{j}^{J}=\Phi_{0}^{2} / 4 \pi^{2} E_{j}^{J}$ the effective inductance of the transmon SQUID at $(j,+)$. A low voltage node is thus created for each transmon qubit (Fig. 1(c)), and the neighboring nodes are connected by coupling SQUIDs with tunable Josephson inductances $L_{j}^{\prime}, \bar{L}_{j}^{\prime} \simeq L_{j}^{J} / 4$. Moreover, the capacitances of the coupling SQUIDs is chosen to be much smaller than $C_{j}$.

The inter-qubit coupling can be established by the current dividing mechanism. An excitation current from the $(j,+)$ qubit which can be written as $l_{j} \simeq \sqrt{\Omega / 2 L_{j}^{j}}\left(b_{j}^{\dagger}+b_{j}\right)$ will mostly flow through $L_{j}^{D}$ to the ground, with a small fractions $l_{j+, j-}$ and $l_{j+,(j-1)-}$ flowing to the neighboring qubits $(j-1,-)$ and $(j,-)$ through the two coupling SQUIDs. The current $l_{j+, j-}$ in turn generates a flux $\Phi_{j+, j-}=\bar{L}_{j}^{D} l_{j+, j-}$ in the $(j,-)$ qubit. The interaction between the two qubits can thus be written as

$H_{j}^{L}=V_{j}\left(b_{j}^{\dagger}+b_{j}\right)\left(\bar{b}_{j}^{\dagger}+\bar{b}_{j}\right)$,

where the coupling constant $V_{j}$ can be estimated as $V_{j} \simeq-\left(\Omega \bar{\Omega} / L_{j}^{J} \bar{L}_{j}^{J}\right)^{1 / 2} \bar{L}_{j}^{D} L_{j}^{D} / L_{j}^{\prime}$. Therefore, the a.c. modulation of the penetrating flux bias in the coupling SQUID loop results in the oscillation of $L_{j}^{\prime}$ and in turn the oscillation of the inter-transmon coupling. Here we should notice that the modulation frequencies of the coupling SQUIDs should not be higher than their plasma frequencies, otherwise complicated quasiparticle excitations would occur. As being mentioned previously, the maximal modulating frequency of the DDM approach is of the order $\varepsilon=\Omega+\bar{\Omega}$. Therefore, with parameters being chosen before, this plasma frequency requirement is guaranteed by the small capacitance of the coupling SQUIDs.

The d.c. bias of the coupling SQUIDs leads to a static nearest neighbor qubit-qubit couplings which is estimated to be on the level of $2 \pi[75,100] \mathrm{MHz}$. Since such nearest-neighbor static coupling strength is much smaller than the energy difference between neighboring qubits (on the level of $2 \pi \times 2.5 \mathrm{GHz}$ ), its influence is the corrections of the hopping and pairing parameters derived from the DDM method. The couplings beyond the nearest neighbors should also be estimated: we notice that the divided current $l_{j+, j-}$ from $(j,+)$ qubit can be further divided in the $(j,-)$ node to flow through the $(j+1,+)$ node, thus the next-nearestneighbor (NNN) $(j,+) \leftrightarrow(j+1,+)$ static coupling is induced. Meanwhile, due to the current dividing mechanism at the lowvoltage nodes, the non-nearest-neighbor couplings decay exponentially with respect to the site distance. The NNN coupling is estimated to be of the order $2 \pi[15,20] \mathrm{MHz}$. To suppress its effect we can use the two-sublattice stragegy for each of the two HCB legs the eigenfrequencies of the four qubits shown in Fig. 1(c) can be modified to be $2 \pi(7.5,10,7,10.5) \mathrm{GHz}$, respectively. Such modification dose not influence the performance of the DDM method as we merely need to adjust the modulating frequencies of $V_{j}(t)$ and $\bar{V}_{j}(t)$ accordingly. However, the effect of the d.c. NNN coupling is significantly suppressed by the $0.5 \mathrm{GHz}$ energy difference between the NNN transmon qubits.

Based on the static bias, we can add the a.c. modulation pluses on the coupling SQUIDs with $s, q, \bar{s}, \bar{q}$ at the order of $2 \pi[10,15] \mathrm{MHz}$ and choose the dispersive active region as $\eta / 2 \pi=75 \mathrm{MHz}$. The resulting dispersive tunneling is estimated to be on the level $w / 2 \pi$, $\Delta / 2 \pi \in[1,5] \mathrm{MHz}$ which is three orders larger than the reported decoherence rates of the transmon qubits (in the range $2 \pi[1,10]$ $\mathrm{kHz}){ }^{33},{ }^{34}$ Such strong tunnel/pairing allow us to set the slow varying envelopes on the level $B_{x}(t) / 2 \pi, B_{y}(t) / 2 \pi, s_{M} / 2 \pi \in[0.05,0.5]$ $\mathrm{MHz}$. Following this setting, the envelopes are all much smaller than the realized bulk gap such that the high energy excitations can be omitted, but stronger enough than the decoherence rates such that the proposed topological quantum operations can be simulated.

\section{DISCUSSION}

The finite anharmonicity of the transmon qubits

Here we should notice that the proposed superconducting transmon qubits are not ideal HCBs but anharmonic oscillators. The assumption of regarding them as HCBs with infinite anharmonicity thus needs further investigation. We recall that the anharmonicity of a transmon qubit is defined by its $\omega_{21}-\omega_{10}{ }^{29}$ and equals its charging energy $E^{C}$, which is at the level of $2 \pi[0.1,0.8] \mathrm{GHz}$. The requirement $E^{J} / E^{C} \gg 1$ should be fulfilled because the qubit becomes more and more sensitive to the background $1 / f$ charge noise with decreasing $E^{J} / E^{C}$, leading to severe dephasing of the physical qubit. ${ }^{29}$ Here we set $E^{C} / 2 \pi=$ $0.75 \mathrm{GHz}$ such that $E^{J} / E^{C}>10$ (previously reported high coherence transmon qubits often work in the region $\left.E^{J} / E^{C} \approx 20\right) .{ }^{31,33}$ Being larger than the dispersive threshold $\eta / 2 \pi=75 \mathrm{MHz}$ by one order of magnitude, such anharmonicity choice prevents the effective excitation of the higher levels of the transmons.

To evaluate the consequence of the finite anharmonictiy, we incorporate the higher levels of the transmon qubits into the ideal $\mathrm{HCB}$ model by enlarging the Hilbert space of each HCB to three dimension, i. e. from $\operatorname{span}\left\{|0\rangle_{j, \bar{j}},|1\rangle_{j, \bar{j}}\right\}$ to $\operatorname{span}\left\{|0\rangle_{j, \bar{j}},|1\rangle_{j, \bar{j}},|2\rangle_{j, j}\right\}$. Following the same essentials of the DDM method, we come to the result that the inclusion of the anharmonicity leads to parasitic terms of the proposed ideal Hamiltonian, taking the form

$$
\begin{aligned}
H_{\text {para }} & =\sum_{j}\left(-w_{\text {para }} \bar{Q}_{j} b_{j}^{\dagger} b_{j+1}+\Delta_{\text {para }} \bar{Q}_{j} b_{j+1} b_{j}+\text { h.c. }\right), \\
\bar{H}_{\text {para }} & =\sum_{j}\left(-\bar{W}_{\text {para }} Q_{j+1} \bar{b}_{j}^{\dagger} \bar{b}_{j+1}-\bar{\Delta}_{\text {para }} Q_{j+1} \bar{b}_{j+1} \bar{b}_{j}+\text { h.c. }\right),
\end{aligned}
$$

where the strengths of these parasitic terms are of the order

$$
\left|w_{\text {para }}\right|,\left|\bar{w}_{\text {para }}\right| \simeq s \bar{s} / E^{C} \ll w=s \bar{s} / \eta,
$$

$\left|\Delta_{\text {para }}\right|,\left|\bar{\Delta}_{\text {para }}\right| \simeq s \bar{q} / E^{C} \ll \Delta=s \bar{q} / \eta$,

and

$Q_{j}=\exp \left(-i \pi c_{j}^{\dagger} c_{j}\right), \bar{Q}_{j}=\exp \left(-i \pi \bar{c}_{j}^{\dagger} \bar{c}_{j}\right)$

with $c_{j}=|1\rangle_{j}\langle 2|$ and $c_{j}^{\dagger}=|2\rangle_{j}\langle 1|$. Moreover, as the excitation to the states $|2\rangle_{j, j}$ is effectively not excited, we can reduce the obtained parasitic terms back to the original 2D HCB Hilbert space 
and get

$$
H_{\text {para }}=\sum_{j}\left[w_{\text {para }}\left(\frac{1}{2}+\frac{1}{2} \bar{P}_{j}\right) b_{j}^{\dagger} b_{j+1}-\Delta_{\text {para }}\left(\frac{1}{2}+\frac{1}{2} \bar{P}_{j}\right) b_{j+1} b_{j}+\text { h.c. }\right] \text {, }
$$

$$
\bar{H}_{\text {para }}=\sum_{j}\left[\bar{W}_{\text {para }}\left(\frac{1}{2}+\frac{1}{2} P_{j+1}\right) \bar{b}_{j}^{\dagger} \bar{b}_{j+1}+\bar{\Delta}_{\text {para }}\left(\frac{1}{2}+\frac{1}{2} P_{j+1}\right) \bar{b}_{j+1} \bar{b}_{j}+\text { h.c. }\right] .
$$

We then observe that the parasitic terms contain two types. The first type containing the $U(1)$ gage factors slightly shift the proposed ideal hopping and pairing constants. This term can be compensated by the refined choice of the pumping parameters. The second type that not containing the $U(1)$ gage factors can be regarded as local perturbation terms. As estimated before, both the two terms have strength smaller than the proposed band gap by one order of magnitude. Therefore, these parasitic terms results in only the slight correction of the ideal scheme. They cannot destroy the performance of the proposed scheme because they are too small to change the band topology of the system by closing and reopening the gap.

\section{Robustness against imperfection factors}

In realistic experiments, the imperfection accompanies inescapably with the proposed ideal scheme. The deviation of the circuit parameters from their ideal values can be attributed to the errors happened in the fabrication process and the low-frequency noises in the proposed circuit. For the fabrication errors, they cause the deviation of the lowest level splitting $\Omega_{j}=\sqrt{8 E_{j}^{C} E_{j}^{j}}$ of the transmons and the current dividing ratios between neighboring transmons from their proposed values. In recent experiments the fabrication-induced disorder in SQC lattice systems has been significantly suppressed by the developing technology. ${ }^{35}$ If the fabrication errors are not too large such that the resulted level splits and current dividing ratios are still around their ideal values with small disorder, they can be corrected by simple refinement of the proposed DDM scheme: The deviation of the level splitting can be compensated by the modification of the frequency of the DDM pulses, and the errors of the current dividing ratio can be compensated by the renormalization of the amplitude of the DDM pulses. ${ }^{36-38}$

The low-frequency $1 / f$ noises can also induce fluctuations of the circuit parameters. ${ }^{39}$ Due to their low frequency property, we can treat the $1 / f$ noises as quasi-static, i. e. the noises do not vary during a experimental run, but vary between different runs. As the proposed HCB chain considered consists of only transmon qubits and coupling SQUIDs with very small charging energies, it is insensitive to the charge type $1 / f$ noise. Such insensitivity has already been extensively investigated in Ref. 29. For the flux $1 / f$ noise penetrated in the loops of the SQUIDs, we notice that previous experiments have shown that the flux noise $\delta \Phi$ in a SQUID loop does not vary greatly with the loop size, inductor value, or temperature, and its strength falls in the range $\delta \Phi / \Phi_{0} \in\left[10^{-6}, 10^{-5}\right] .^{40-43}$ The consequent fluctuation effects can then be estimated as

$\delta \Omega_{j} / 2 \pi<10^{-1} \mathrm{MHz}, \quad \delta s_{j}, \delta q_{j}<10^{-3} \mathrm{MHz}$,

where both of which induce local perturbation to the ideal Hamiltonian with amplitudes much smaller than the proposed band gap of the DIII chain. Such small fluctuations cannot destroy the MZMs as they are too small to change the band topology by closing and reopening the gap (notice that this argument applies to the other parameter fluctuations, and such robustness roots from the topological nature of our scheme). In addition, experiments have shown that the influence of the critical current noise in a large Josephson junction is even smaller than those of the flux $1 / f$ noise and can then be safely neglected. ${ }^{42}$ Summarizing the analysis above, we then come to the conclusion that our scheme can survive in the presence of the $1 / f$ noises in SQC devices.

\section{CONCLUSION}

In conclusion, we have designed a topolgy-preseved HCB simulator for the TRS-invariant DIII topological superconducting chains and proposed a set of universal quantum gates with topological bases through the developed DDM technique. Physical implementation of our proposal with SQCs has also been explored. The present results may pave the way for realizing universal quantum computation with topological stability.

\section{METHODS}

The derivation of the DDM pulses

Here we detail the derivation of DDM pulses and the consequent effective Hamiltonian. We exploit the dispersive coupling mechanism which states that, for a system governed by a fast oscillating Hamiltonian $A e^{i \omega t}+B e^{-i \omega t}$ with $\|A\|,\|B\| \ll \omega$, its evolution can be described by the effective Hamiltonian $H^{E E f}=[A, B] / \omega^{44,45}$ (here the norm $\|O\|$ of an operator $O$ is defined as the square root of the largest eigenvalue of $O^{\dagger} O$ ). Our basic idea is that, if we are able to use the $A=b_{j}^{\dagger} \bar{b}_{j}$ term in $\mathcal{H}_{j}^{L}$ as $A$ and the $\bar{b}_{j}^{\dagger} b_{j+1}^{\dagger}$ and $\bar{b}_{j}^{\prime} b_{j+1}$ terms in $\overline{\mathcal{H}}_{j}^{L}$ as $B$, the resulting commutators $[A, B]$ provide the required $b_{j}^{\dagger} b_{j+1}^{\dagger} \bar{P}_{j}$ and $b_{j}^{\dagger} b_{j+1} \bar{P}_{j}$ terms. For this purpose we adopt the rotating frame for which $\mathcal{H}_{j}^{L}$ and $\overline{\mathcal{H}}_{j}^{L}$ take the forms

$\mathcal{H}_{j}^{L}=V_{j}\left(b_{j}^{\dagger} \bar{b}_{j}^{\dagger} e^{i \varepsilon t}+b_{j}^{\dagger} \bar{b}_{j} e^{i \delta t}+b_{j} \bar{b}_{j}^{\dagger} e^{-i \delta t}+b_{j} \bar{b}_{j} e^{-i \varepsilon t}\right)$,

$\overline{\mathcal{H}}_{j}^{L}=\bar{V}_{j}\left(\bar{b}_{j}^{\dagger} b_{j+1}^{\dagger} e^{i \varepsilon t}+\bar{b}_{j}^{\dagger} b_{j+1} e^{-i \delta t}+\bar{b}_{j} b_{j+1}^{\dagger} e^{i \delta t}+\bar{b}_{j} b_{j+1} e^{-i \varepsilon t}\right)$.

As being noticed already, the four terms in $\mathcal{H}_{j}^{L}$ and $\overline{\mathcal{H}}_{j}^{L}$ oscillate with frequencies $\varepsilon, \delta,-\delta$ and $-\varepsilon$, respectively. Meanwhile, the dynamic modulation of $V_{j}(t)$ and $\bar{V}_{j}(t)$ can synthesize the dispersive coupling by shifting the frequencies of the terms in Eq. (16) upward and downward. For the modulation $V_{j}(t)=2 s_{j} \cos (\eta-\delta) t+2 q_{j} \cos (\eta-\varepsilon) t$, we find that $\mathcal{H}_{j}^{p}=s_{j} b_{j}^{\dagger} \bar{b}_{j}+q_{j} b_{j}^{\dagger} b_{j}^{\dagger}$ is positively activated (i.e. it oscillates with frequency $\eta)$ and its Hermitian conjugate $\mathcal{H}_{j}^{N}=s_{j} b_{j} \bar{b}_{j}^{\dagger}+q_{j} \bar{b}_{j} b_{j}$ is negatively activated. The other terms in $\mathcal{H}_{j}^{L}$ are de-activated because their frequencies are far away from the dispersive active region $[-\eta, \eta]$ at least by the order of $\delta$. Also, for the modulation $\bar{V}_{j}(t)=2 \bar{s}_{j} \cos (\eta-\delta) t+2 \bar{q}_{j} \cos (\eta+\varepsilon) t$, we can positively activate $\overline{\mathcal{H}}_{j}^{P}=\bar{s}_{j} \bar{b}_{j} b_{j+1}^{\dagger}+\bar{q}_{j} \bar{b}_{j} b_{j+1}$ and negatively activate $\overline{\mathcal{H}}_{j}^{N}=\bar{s}_{j} \bar{b}_{j}^{\dagger} b_{j+1}+\bar{q}_{j} \bar{b}_{j}^{\dagger} b_{j+1}^{\dagger}$. The dispersive coupling between $\mathcal{H}_{j}^{L}$ and $\overline{\mathcal{H}}_{j}^{L}$ results in two effects. The first is the self part

$\left(\left[\mathcal{H}_{j}^{P}, \mathcal{H}_{j}^{N}\right]+\left[\overline{\mathcal{H}}_{j}^{P}, \overline{\mathcal{H}}_{j}^{N}\right]\right) / \eta$

which contains the self-energy corrections of the sites $(j,+),(j,-)$, and $(j+1$, $+)$. The more nontrivial terms emerge from the mutual dispersive coupling

$\left(\left[\mathcal{H}_{j}^{P}, \overline{\mathcal{H}}_{j}^{N}\right]+\left[\overline{\mathcal{H}}_{j}^{P}, \mathcal{H}_{j}^{N}\right]\right) / \eta$

which can be simplified as

$\mathcal{H}_{j}^{\text {Eff }}=\frac{s_{j} \bar{s}_{j}}{\eta} b_{j}^{\dagger} b_{j+1} \bar{P}_{j}+\frac{s_{j} \bar{q}_{j}}{\eta} b_{j}^{\dagger} b_{j+1}^{\dagger} \bar{P}_{j}+$ h.c.

Similarly, the dispersive coupling between the $(j,-) \leftrightarrow(j+1,+)$ and $(j+1$, $+) \leftrightarrow(j+1,-)$ links provides the effective $(j,-) \leftrightarrow(j+1,-)$ coupling

$\overline{\mathcal{H}}_{j}^{E f f}=-\frac{\bar{s}_{j} s_{j+1}}{\eta} \bar{b}_{j}^{\dagger} \bar{b}_{j+1} P_{j+1}-\frac{\bar{s}_{j} q_{j+1}}{\eta} \bar{b}_{j}^{\dagger} \bar{b}_{j+1}^{\dagger} P_{j+1}+$ h.c.

Notice that all the effective hopping and pairing constants in Eqs. (18), (19) can be independently controlled by the pumping parameters $\left(s_{j}, q_{j}\right)$ and $\left(\bar{s}_{j}, \bar{q}_{j}\right)$. Through the setting

$s_{j}=(-1)^{j-1} s, q_{j}=(-1)^{j-1} q$,

$\bar{s}_{j}=(-1)^{j-1} \bar{s}, \bar{q}_{j}=(-1)^{j-1} \bar{q}, \bar{q} / \bar{s}=-q / s$,

the population-dependent phase terms in the bosonic version of $H_{\text {DIII }}$ can be directly reproduced from Eqs. (18), (19). 


\section{ACKNOWLEDGEMENTS}

We thank T. Mao and D. Zhang for helpful discussions. This work was supported in part by the National Science Foundation of China (Grant No. 11374117), the NKRDP of China (Grant No. 2016YFA0301802), the GRF (HKU173051/14P \& HKU173055/15P), the CRF (HKU8/11G) of Hong Kong, the fellowship of HongKong Scholars Program (Grant No. 2012-80), and the National Fundamental Research Program of China (Grant No. 2011CB922104, No. 2012CB922103, and No. 2013CB921804).

\section{AUTHOR CONTRIBUTIONS}

Z.D.W. proposed the idea. Y.H. and Z.Y.X. carried out all calculations under the guidance of Z.D.W. Y.X.Z. participated in the discussions and the interpretation of the work. Y.H., Y.X.Z., and Z.D.W. contributed to the writing of the manuscript.

\section{COMPETING INTERESTS}

The authors declare no competing interests.

\section{REFERENCES}

1. Hasan, M. Z. \& Kane, C. L. Colloquium: topological insulators. Rev. Mod. Phys. 82, 3045-3067 (2010).

2. Qi, X.-L. \& Zhang, S.-C. Topological insulators and superconductors. Rev. Mod. Phys. 83, 1057-1110 (2011).

3. Bernevig, A. B. \& Hughes, T. L. Topological Insulators and Topological Superconductor (Princeton University Press, 2013).

4. Schnyder, A. P., Ryu, S., Furusaki, A. \& Ludwig, A. W. W. Classification of topological insulators and superconductors in three spatial dimensions. Phys. Rev. B 78, 195125 (2008)

5. Kitaev, A. Periodic table for topological insulators and superconductors. AIP Conf. Proc. 1134 (2009).

6. Klitzing, K. V., Dorda, G. \& Pepper, M. New method for high-accuracy determination of the fine-structure constant based on quantized Hall resistance. Phys. Rev. Lett. 45, 494-497 (1980).

7. Kitaev, A. Y. Unpaired Majorana fermions in quantum wires. Phys. Usp 44, 131 (2001).

8. Wong, C. L. M. \& Law, K. T. Majorana Kramers doublets in -wave superconductors with Rashba spin-orbit coupling. Phys. Rev. B 86, 184516 (2012).

9. Nakosai, S., Budich, J. C., Tanaka, Y., Trauzettel, B. \& Nagaosa, N. Majorana bound states and nonlocal spin correlations in a quantum wire on an unconventional superconductor. Phys. Rev. Lett. 110, 117002 (2013).

10. Keselman, A., Fu, L., Stern, A. \& Berg, E. Inducing time-reversal-invariant topological superconductivity and fermion parity pumping in quantum wires. Phys. Rev. Lett. 111, 116402 (2013).

11. Zhang, F., Kane, C. L. \& Mele, E. J. Topological mirror superconductivity. Phys. Rev. Lett. 111, 056403 (2013).

12. Zhao, Y. X. \& Wang, Z. D. Exotic topological types of Majorana zero modes and their universal quantum manipulation. Phys. Rev. B 90, 115158 (2014).

13. Dumitrescu, E., Sau, J. D. \& Tewari, S. Magnetic field response and chiral symmetry of time-reversal-invariant topological superconductors. Phys. Rev. B 90, 245438 (2014).

14. Gaidamauskas, E., Paaske, J. \& Flensberg, K. Majorana bound states in twochannel time-reversal-symmetric nanowire systems. Phys. Rev. Lett. 112, 126402 (2014).

15. Zhao, Y. X. \& Wang, Z. D. Topological classification and stability of Fermi surfaces. Phys. Rev. Lett. 110, 240404 (2013).

16. Zhao, Y. X. \& Wang, Z. D. Topological connection between the stability of Fermi surfaces and topological insulators and superconductors. Phys. Rev. B 89, 075111 (2014).

17. Zhao, Y. X. \& Wang, Z. D. Novel $Z_{2}$ topological metals and semimetals. Phys. Rev. Lett. 116, 016401 (2016).

18. Carusotto, l. et al. Fermionized photons in an array of driven dissipative nonlinear cavities. Phys. Rev. Lett. 103, 033601 (2009).

19. Bardyn, C.-E. \& Imamoğlu, A. Majorana-like modes of light in a one-dimensional array of nonlinear cavities. Phys. Rev. Lett. 109, 253606 (2012).

20. You, J. Q., Wang, Z. D., Zhang, W. \& Nori, F. Encoding a qubit with Majorana modes in superconducting circuits. Sci. Rep. 4, 5535 (2014).
21. Mao, T. \& Wang, Z. D. Quantum simulation of topological Majorana bound states and their universal quantum operations using charge-qubit arrays. Phys. Rev. A 91, 012336 (2015).

22. Marcos, D., Rabl, P., Rico, E. \& Zoller, P. Superconducting circuits for quantum simulation of dynamical gauge fields. Phys. Rev. Lett. 111, 110504 (2013).

23. Garca-Álvarez, L. et al. Fermion-fermion scattering in quantum field theory with superconducting circuits. Phys. Rev. Lett. 114, 070502 (2015).

24. Zhu, S.-L., Zhang, D.-W. \& Wang, Z. D. Delocalization of relativistic Dirac particles in disordered one-dimensional systems and its implementation with cold atoms. Phys. Rev. Lett. 102, 210403 (2009).

25. You, J. Q. \& Nori, F. Atomic physics and quantum optics using superconducting circuits. Nature. 474, 589-597 (2011).

26. Devoret, M. H. \& Schoelkopf, R. J. Superconducting circuits for quantum information: an outlook. Science 339, 1169-1174 (2013).

27. Houck, A. A., Tureci, H. E. \& Koch, J. On-chip quantum simulation with superconducting circuits. Nat. Phys 8, 292-299 (2012).

28. Schmidt, S. \& Koch, J. Circuit QED lattices: towards quantum simulation with superconducting circuits. Annalen der Physik 525, 395-412 (2013).

29. Koch, J. et al. Charge-insensitive qubit design derived from the Cooper pair box. Phys. Rev. A 76, 042319 (2007).

30. Schreier, J. A. et al. Suppressing charge noise decoherence in superconducting charge qubits. Phys. Rev. B 77, 180502 (2008).

31. Chen, Y. et al. Qubit architecture with high coherence and fast tunable coupling. Phys. Rev. Lett. 113, 220502 (2014).

32. Geller, M. R. et al. Tunable coupler for superconducting Xmon qubits: perturbative nonlinear model. Phys. Rev. A 92, 012320 (2015).

33. Jin, X. Y. et al. Thermal and residual excited-state population in a $3 \mathrm{~d}$ transmon qubit. Phys. Rev. Lett. 114, 240501 (2015).

34. Barends, R. et al. Coherent Josephson qubit suitable for scalable quantum integrated circuits. Phys. Rev. Lett. 111, 080502 (2013).

35. Underwood, D. L., Shanks, W. E., Koch, J. \& Houck, A. A. Low-disorder microwave cavity lattices for quantum simulation with photons. Phys. Rev. A 86, 023837 (2012).

36. Wang, Y. P. et al. Realizing and characterizingchiral photon flow in a circuit quantum electrodynamics necklace. Sci. Rep. 5, 8352 (2015).

37. Wang, Y.-P., Yang, W.-L., Hu, Y., Xue, Z.-Y. \& Wu, Y. Detecting topological phases of microwave photons in a circuit quantum electrodynamics lattice. npj Quantum Inf. 2, 16015 (2016).

38. Yang, Z.-H. et al. Circuit quantum electrodynamics simulator of flat band physics in a Lieb lattice. Phys. Rev. A 93, 062319 (2016).

39. Paladino, E., Galperin, Y. M., Falci, G. \& Altshuler, B. L. 1/f noise: Implications for solid-state quantum information. Rev. Mod. Phys. 86, 361-418 (2014).

40. Wellstood, F. C., Urbina, C. \& Clarke, J. Low frequency noise in dc superconducting quantum interference devices below 1 K. Appl. Phys. Lett. 50, 772-774 (1987).

41. Yoshihara, F., Harrabi, K., Niskanen, A. O., Nakamura, Y. \& Tsai, J. S. Decoherence of flux qubits due to $1 / f$ flux noise. Phys. Rev. Lett. 97, 167001 (2006).

42. Bialczak, R. C. et al. 1/f flux noise in Josephson phase qubits. Phys. Rev. Lett. 99, 187006 (2007).

43. Lanting, T. et al. Geometrical dependence of the low-frequency noise in superconducting flux qubits. Phys. Rev. B 79, 060509 (2009).

44. Goldman, N. \& Dalibard, J. Periodically driven quantum systems: effective hamiltonians and engineered gauge fields. Phys. Rev. X 4, 031027 (2014).

45. Goldman, N., Dalibard, J., Aidelsburger, M. \& Cooper, N. R. Periodically driven quantum matter: the case of resonant modulations. Phys. Rev. A 91, 033632 (2015).

This work is licensed under a Creative Commons Attribution 4.0 International License. The images or other third party material in this article are included in the article's Creative Commons license, unless indicated otherwise in the credit line; if the material is not included under the Creative Commons license, users will need to obtain permission from the license holder to reproduce the material. To view a copy of this license, visit http://creativecommons.org/licenses/by/ 4.0/

(c) The Author(s) 2017 